\title{
Revista de Psicología Clínica con Niños y Adolescentes
}

\section{-Funcionamiento familiar en adolescentes en riesgo de suicidio con rasgos de personalidad límite: un estudio exploratorio}

\author{
Sara Marco-Sánchez ${ }^{1}$, María Mayoral-Aragón ${ }^{2}$, Fátima Valencia-Agudo ${ }^{3}$, Laura Roldán-Díaz ${ }^{2}$, Ana Espliego- \\ Felipe $^{2}$, Carlos Delgado-Lacosta ${ }^{2}$, \& Gonzalo Hervás-Torres ${ }^{3}$ \\ ${ }^{1}$ Hospital Sant Joan de Déu Terres de Lleida, España \\ ${ }^{2}$ Hospital General Universitario Gregorio Marañón, España \\ ${ }^{3}$ Universidad Complutense de Madrid, España.
}

\begin{abstract}
Resumen
La alta prevalencia de suicidio en población adolescente en todo el mundo supone un reto mundial en salud mental. El ambiente familiar ha sido señalado como una importante variable implicada en el riesgo de suicidio de los adolescentes, ejerciendo tanto de factor de riesgo como protector. El estudio tiene como objetivo entender qué variables del funcionamiento familiar de la Escala de Clima Social en la Familia se relacionan con la puntuación de la Escala de Ideación Suicida de Beck en una muestra 37 adolescentes entre 14 y 18 años $(M=15.76 ; D T=1.07)$ con rasgos de personalidad límite. Se partió de la hipótesis de que peores niveles de funcionamiento familiar aumentarían el riesgo de suicidio. Los resultados mostraron que la ideación suicida era mayor cuando las puntuaciones en la escala Relaciones (calidad de la comunicación e interacción) en la familia eran menores. Al analizar cada escala, se demostró la importancia especifica de la subescala Expresividad, referida al grado de expresión libre de los sentimientos en la familia de forma que, a menor expresividad en la familia, mayor ideación suicida presentaban los adolescentes, y la subescala Social-Recreativa, referida a la participación social y en actividades de ocio de la familia, de modo que a mayor participación social, menor riesgo suicida. Estos resultados son apoyados por otros estudios que demuestran la importancia de modificar el entorno familiar para influir en el riesgo suicida de los adolescentes. El valor de estos datos implica la consideración de incluir a las familias en los programas de tratamiento para adolescentes suicidas.
\end{abstract}

Palabras clave: suicidio; trastorno límite; factor de riesgo; familias; adolescentes.

\begin{abstract}
Family functioning in suicidal adolescents with borderline personality traits. High prevalence of suicide in adolescent population all over the world is considered a world challenge in mental health. Family environment has been pointed as an important factor involved in adolescents' suicide risk, playing a role not only as a risk factor but also as protective. This study has its goal on understanding which factors of the family functioning, measured by the Social Environment in the Family Scale, are related to the total score of the Beck Suicide Ideation Scale in a sample of 37 adolescents with ages between 14 and 17 years old $(M=15.76 ; S D=1.06)$ borderline personality traits. It was hypothesized that worse levels of family functioning will increase suicide risk in adolescents. Results showed that the risk of suicide was higher when the Relationships Scale total score (quality of communication and interaction in the family) was lower. After analyzing each subscale separately, it was demonstrated the specific relevance of Expressivity Subscale, referred to the level of free expression of feelings in the family, suggesting that the lower expressivity in the family, the higher suicide ideation showed by the adolescents, and Social-Recreative, referred to the social participation and leisure of the family, what means the more social participation, the less suicide ideation. These results are supported by other studies that show the relevance of modifying the family environment for reducing the suicidal risk among adolescents. The value of this data involves taking into account the families in the treatment programs for suicidal adolescents.
\end{abstract}

Keywords: suicide; borderline personality disorder; risk factor; families; adolescents.

La prevalencia de suicidio en población adolescente en todo el mundo supone un reto mundial en salud mental. Según datos de la OMS (2014), el suicidio es la segunda causa de muerte en población entre 15 y 29 años. Uno de los principales factores de riesgo de suicidio es la presencia de un trastorno mental o trastorno de la personalidad, especialmente el 
trastorno límite de la personalidad (Consoli et al., 2015; Miller, Rathus, \& Linehan, 2007) debido a la alta inestabilidad emocional que presentan las personas que lo padecen y a sus dificultades en la regulación y el manejo de las emociones.

El trastorno límite de personalidad (TLP) es un trastorno mental grave comúnmente asociado a discapacidades funcionales severas y a un alto riesgo de suicidio (Kaess, Brunner, \& Chanen, 2014). Se trata de una forma de psicopatología caracterizada por un constante patrón de inestabilidad en la regulación emocional, en el control de impulsos, las relaciones interpersonales y el sentido de uno mismo (American Psychiatric Association, 2013). Investigaciones recientes demuestran que el diagnóstico de TLP es fiable y válido entre adolescentes como lo es en adultos, y que los adolescentes con TLP pueden beneficiarse de intervenciones terapéuticas tempranas (Conway, Hammen, \& Brennan, 2015; Miller, Muehlenkamp, \& Jacobson, 2008; Yen, Gagnon, \& Spirito, 2013). Asimismo, el DSM 5 (APA, 2013) y la CIE 11 (Tyrer, Crawford, \& Mulder, 2011) han confirmado la legitimidad del diagnóstico de TLP en adolescentes (Kaess et al., 2014).

Linehan (1993) postula que el núcleo del TLP es la desregulación emocional, la cual es desarrollada y mantenida por una transacción entre una vulnerabilidad biológica y un entorno invalidante. La vulnerabilidad biológica se expresa en una alta sensibilidad a los estímulos emocionales, una respuesta emocional intensa y un retorno lento a la línea base emocional. Por su parte, el entorno invalidante sería aquel que negase, castigase o respondiera erráticamente o inapropiadamente a las experiencias privadas, reforzando intermitentemente la escalada emocional y sobresimplificando la facilidad de la resolución de los problemas. Como consecuencia, estos individuos nunca aprenden a etiquetar y regular las emociones, tolerar el estrés o confiar en sus propias respuestas emocionales. Siguiendo los postulados de la teoría de Linehan, una de las estrategias destinadas a reducir el riesgo suicida en adolescentes se basa en influir en el medio que les rodea, principalmente la familia.

Otros autores también han considerado la relevancia del ambiente intrafamiliar en el riesgo de suicidio en adolescentes. Xing et al. (2010) encontraron una fuerte asociación entre estar expuesto a ambientes familiares disfuncionales y los intentos de suicidio en adolescentes. Estos autores señalan la importancia del clima familiar, el nivel de conflicto y la pobre comunicación como factores de riesgo para las conductas autolesivas y suicidas en adolescentes. Machell, Rallis, y Esposito-Smythers (2016) encontraron resultados similares en una muestra de adolescentes con ansiedad, de modo que la ideación suicida aumentaba cuando los adolescentes percibían un bajo apoyo familiar.

El ambiente familiar también ha sido considerado como factor protector de suicidio en adolescentes, especialmente la variable referida al apoyo familiar (Compton, Thompson, \& Kaslow, 2005; Meadows, Kaslow, \& Skegg, 2005). La relación entre los comportamientos suicidas y las relaciones familiares se da de forma bidireccional, ya que estos comportamientos actúan como fuertes estresores en la familia (Lucksted, Stewart, \& Forbes, 2008) aumentando el grado de conflicto y de vulnerabilidad del sistema familiar. Los comportamientos autolesivos y suicidas impactan a todo el sistema familiar, interfiriendo en la dinámica y el funcionamiento de la fami- lia. Los miembros de la familia son habitualmente con quienes los adolescentes comparten sus pensamientos suicidas, quienes suelen intervenir tras el intento de suicidio y quienes se implican durante el ingreso hospitalario (Fulginiti, Rice, Hsu, Rhoades, \& Winetrobe, 2016).

Las familias de los pacientes con riesgo suicida tienden a sentirse desbordados debido a la preocupación y el miedo que genera la posibilidad de que sus hijos se suiciden (McDonell, Short, Berry, \& Dyck, 2003). Los padres expresan una necesidad de apoyo, información y habilidades para manejar la relación con sus hijos, mejorar su comunicación y en cómo actuar con sus hijos cuando se autolesionan o tratan de suicidarse (Raphael, Clarke, \& Cumar, 2006).

El objetivo de este estudio es conocer cuáles son los componentes concretos del funcionamiento familiar que se asocian a un mayor riesgo de suicidio en una muestra de adolescentes con rasgos de personalidad límite. Siguiendo a los resultados de estudios previos, esperamos que las variables relativas al apoyo familiar ejerzan un papel de importante en el riesgo suicida de los adolescentes, de modo que un menor apoyo familiar percibido se relacione con un mayor riesgo de suicidio en los hijos.

\section{Método}

\section{Participantes}

Los participantes fueron 37 adolescentes, todos ellos cumplían criterios de trastorno límite de la personalidad evaluado a través de la entrevista SCID-II aplicada por psicólogos clínicos y psiquiatras con experiencia en población psiquiátrica infanto-juvenil. La muestra estaba compuesta por 35 mujeres y 2 varones, siendo el rango de edades entre 14 y 17 años, $(M=15.76 ; D T=1.06)$. El $78.4 \%$ era de origen español, el $16.2 \%$ de países de Latinoamérica y el $5.4 \%$ de otras nacionalidades. En cuanto al rendimiento académico, el $48.6 \%$ presentaba fracaso escolar, otro $48.6 \%$ un rendimiento medio y un $2.8 \%$ presentaba un rendimiento sobresaliente. Respecto al nivel socioeconómico, medido a través de la escala Hollinhead-Rendlich, el $10.8 \%$ pertenecían al nivel II, el 32.4\% al nivel III, el 37.8 al nivel IV y el 18.9 al nivel V. La mayoría de ellos (81\%) presentaba comorbilidad con un diagnóstico del Eje I, siendo el más prevalente el de trastorno depresivo mayor. El 92\% había presentado autolesiones a lo largo de su vida, mientras que el 59\% lo había hecho durante el último mes. El 69\% de la muestra había realizado algún intento de suicidio a lo largo de su vida y todos ellos habían presentado ideación suicida en los últimos tres meses.

Los criterios de inclusión fueron los siguientes: 1) edad del paciente entre 14-18 años; 2) haber sufrido un intento, gesto suicida o ideación suicida persistente en los últimos 6 meses; y 3) cumplir al menos tres de los nueve criterios requeridos para el diagnóstico de trastorno límite de la personalidad (DSM5, APA 2013). Los criterios de exclusión consistieron en: 1) presencia de retraso mental; 2) cumplimiento de criterios diagnósticos para algún trastorno psiquiátrico que incapacitara el aprendizaje de habilidades; 3) existencia de abuso de sustancias como diagnóstico primario; y 4) no conseguir el consentimiento informado. 


\section{Instrumentos}

Los participantes fueron evaluados mediante las entrevistas diagnósticas Kiddie Schedule for Affective Disorders and Schizophrenia (KSADS; Chambers et al., 1985) y la Entrevista Clínica Estructurada para los Trastornos de la Personalidad del Eje II del DSM-IV (SCID-II; First et al., 1997) para confirmar su inclusión en el programa. Fueron también recogidos los datos de hospitalizaciones, intentos de suicidio previos y autolesiones no suicidas entre otros.

\section{Fueron administrados los siguientes cuestionarios:}

Escala de Ideación Suicida de Beck (Beck, Kovacs, \& Weissman, 1979). Se trata de un cuestionario para evaluar y cuantificar la intencionalidad suicida, o grado de seriedad e intensidad con el que alguien ha pensado en suicidarse en la última semana. Es una medida de autoinforme empleada en adultos y adolescentes que consta de 21 ítems que recogen características relativas a la actitud hacia la vida y la muerte, los pensamientos o deseos suicidas, el proyecto de intento de suicidio y la realización del intento de suicidio. Para cada ítem hay tres alternativas de respuesta en una escala Likert que indican un grado creciente de seriedad e intensidad de la intencionalidad suicida. Los dos últimos ítems indagan sobre los antecedentes previos de suicidio, que no se contabilizan en la puntuación total. La puntuación puede ir de 19 a 57, siendo mayor la ideación suicida conforme mayor es la puntuación. El estudio de fiabilidad mostró una consistencia interna alta (coeficiente de alfa de Cronbach de .89) y una fiabilidad inter examinador de .83. Presenta una excelente consistencia y validez de contenido, constructo y concurrente. En cuanto a validez, su correlación con la Escala de Desesperanza de Beck fue de .75 y con la Escala para la Evaluación de la Depresión de Hamilton de.30.

Tabla 1. Dimensiones y subescalas de la Escala de Clima Social Familiar

\begin{tabular}{|c|c|}
\hline Dimensiones & Subescalas \\
\hline Relaciones: grado & Expresividad: grado en que se anima a los \\
\hline de comunicación & miembros a actuar libremente y a expresar \\
\hline y libre expresión & directamente sus sentimientos. \\
\hline dentro de la & Cohesión: grado en que los miembros de la familia \\
\hline familia y el grado & están compenetrados y se ayudan y se apoyan entre sí. \\
\hline de interacción & Conflicto: grado en que se expresan libre y \\
\hline conflictiva que la & abiertamente la cólera, agresividad y conflicto entre \\
\hline caracteriza. & los miembros de la familia. \\
\hline Desarrollo: & Autonomía: grado en que los miembros de \\
\hline importancia & la familia están seguros de sí mismos, son \\
\hline que tienen & autosuficientes y toman sus propias decisiones. \\
\hline en la familia & Actuación: grado en que las actividades (tales como \\
\hline los procesos & escuela o trabajo) se enmarcan en una estructura \\
\hline de desarrollo & orientada a la acción o competitiva \\
\hline personal, & Social-recreativa: grado de participación en \\
\hline fomentados o no & actividades sociales y recreativas. \\
\hline en la familia. & $\begin{array}{l}\text { Moralidad-Religiosidad: importancia que se da a las } \\
\text { prácticas y valores de tipo ético y religioso. } \\
\text { Intelectual-Cultural: grado de interés en actividades } \\
\text { políticas, sociales, intelectuales y culturales }\end{array}$ \\
\hline Estabilidad: & Organización: importancia que se da a una \\
\hline estructura y & clara organización y estructura al planificar las \\
\hline organización de la & actividades y responsabilidades de la familia. \\
\hline familia y grado de & Control: grado en que la dirección de la vida \\
\hline control que unos & familiar se atiene a reglas y procedimientos \\
\hline $\begin{array}{l}\text { miembros ejercen } \\
\text { sobre otros. }\end{array}$ & establecidos. \\
\hline
\end{tabular}

Escala de Clima Social en la Familia (Moos, 1974; adaptación española de Seisdedos, De la Cruz, \& Cordero, 1984). Esta escala se dirige a conocer las características sociofamiliares, evaluando las relaciones interpersonales entre los miembros de la familia, los aspectos de desarrollo que tienen mayor importancia para ella y su estructura básica. Está constituida por 90 ítems, agrupadas en 10 subescalas que definen tres dimensiones fundamentales: relaciones, desarrollo y estabilidad (Tabla 1). En los estudios originales (Insel \& Moos, 1974), las fiabilidades de las diferentes subescalas del FES estaban comprendidas entre .61 y .78. El análisis de consistencia interna sugiere que las subescalas miden aspectos relativamente diferentes del clima familiar pero dentro de una consistencia considerable. La validez discriminante ha sido demostrada para diferenciar entre familias funcionales y disfuncionales.

\section{Procedimiento}

Los participantes fueron evaluados como candidatos al programa Acciones para el Tratamiento de la Personalidad en la Adolescencia (ATraPA@) de la Unidad de Hospitalización Aguda de Adolescentes (UADO) del Hospital General Universitario Gregorio Marañón (Madrid).

\section{Análisis estadísticos}

En primer lugar, se determinaron los estadísticos descriptivos de las puntuaciones de las subescalas de la Escala de Clima Social en la Familia.

Tabla 2. Estadísticos descriptivos de las subescalas de la Escala de Clima Social en la Familia

\begin{tabular}{|lcc|}
\hline & $M$ & $D T$ \\
\hline Cohesión & 3.65 & 2.43 \\
Expresividad & 3.68 & 3 \\
Conflicto & 5.79 & 2.52 \\
Autonomía & 5.16 & 1.81 \\
Actuación & 5.09 & 2.36 \\
Social-recreativa & 4.31 & 2.21 \\
Intelectual-cultural & $4, .00$ & 2.52 \\
Moral-religiosa & 2.79 & 1.68 \\
Organización & 4.70 & 2.67 \\
Control & 4.21 & 1.99 \\
\hline
\end{tabular}

La normalidad de la distribución de datos se determinó mediante la prueba Shapiro-Wilk. Se llevó a cabo un análisis de correlaciones de Pearson entre las diferentes dimensiones del Cuestionario de Clima Social en la Familia y la puntuación global del Cuestionario de Ideación Suicida. Posteriormente, se llevó a cabo un análisis de correlaciones de Pearson entre la Ideación Suicida y las subescalas de las dimensiones que se relacionaban de manera estadísticamente significativa con la Ideación suicida. Finalmente, se llevó a cabo un análisis de regresión lineal, donde las variables independientes fueron de las tres dimensiones del Cuestionario de Clima Social en la Familia y la variable dependiente la puntuación total de la Escala de Ideación Suicida. Los análisis estadísticos se llevaron a cabo con el programa IBM SPSS Statistics v. 22. 


\section{Resultados}

Los resultados de los análisis de correlación entre las dimensiones de la Escala Clima Social en la Familia y la Escala de Ideación Suicida se muestran en la Tabla 2. Se observó una correlación indirecta entre la dimensión Relaciones de la Escala de Clima Social en la Familia y la puntuación total de la Escala de Ideación Suicida $(r=-.59 ; p<.05)$. Las dimensiones Desarrollo y Estabilidad presentaron ambas correlaciones negativas con la Escala de Ideación Suicida, pero no fueron significativas.

Tabla 3. Correlaciones entre dimensiones de la Escala Clima Social en la Familia y la puntuación global de la Escala de Ideación Suicida

\begin{tabular}{|lc|}
\hline Dimensiones de la escala Clima Familiar & Ideación suicida \\
\hline Relaciones & $-.59^{\star}$ \\
Desarrollo & -.54 \\
Estabilidad & -.24 \\
\hline
\end{tabular}

${ }^{*} p<.05$

Posteriormente se llevó a cabo un análisis de correlaciones entre las diferentes subescalas de la Escala Clima Social en la Familia y la Escala de Ideación Suicida. Los resultados se muestran en la Tabla 3. Expresividad es la única subescala de la dimensión de Relaciones que muestra una correlación significativa, aunque indirecta, con la Ideación Suicida $(r=-.56 ; p<$ $.05)$, de modo que los participantes con puntuaciones más altas en Expresividad presentaban menor puntuación en la Escala de Ideación Suicida. De las subescalas de la dimensión Desarrollo, la subescala Social-recreativa se relacionó significativamente, aunque de forma indirecta, con la puntuación total de Ideación Suicida $(r=-.55 ; p<.05)$, de manera que los participantes con puntuaciones mayores en la subescala Social-Recreativa presentaron una menor puntuación total en la Escala de Ideación Suicida. Ninguna de las subescalas de la dimensión Estabilidad estuvo estadísticamente relacionada con las puntuaciones de la Escala de Ideación Suicida.

Tabla 4. Correlaciones entre las subescalas de las diferentes dimensiones y la puntuación global de la Escala de Ideación Suicida

\begin{tabular}{|lc|}
\hline Subescalas de la dimensión Relaciones & Ideación suicida \\
\hline Cohesión & -.13 \\
Expresividad & $-.56^{*}$ \\
Conflicto & -.31 \\
Autonomía & .17 \\
Actuación & .17 \\
Intelectual-cultural & -.35 \\
Social-recreativa & $-.55^{\star}$ \\
Moral-religiosa & -.31 \\
Organización & -.22 \\
Control & -.29 \\
\hline
\end{tabular}

$$
{ }^{*} p<.05
$$

Finalmente, se llevó a cabo un análisis de regresión lineal de las dimensiones de la Escala de Ideación Suicida sobre la Escala de Ideación Suicida. Los resultados se muestran en la Tabla 4. Los análisis muestran un valor de $\mathrm{R}^{2}$ de .8 , siendo el $80 \%$ de la varianza de la variable dependiente explicada por el modelo. De las tres subescalas, la única que presenta resul- tados significativos es la de Relaciones, con $p<.05$, por lo que tanto Estabilidad como Desarrollo no fueron buenas variables explicativas de la varianza de las puntuaciones de la Escala de Ideación Suicida.

Tabla 5. Análisis de regresión lineal de las dimensiones de la Escala Clima Social en la Familia sobre la Escala de Ideación Suicida

\begin{tabular}{|c|c|c|c|c|}
\hline \multirow[t]{2}{*}{ Modelo* } & \multicolumn{3}{|c|}{ Coeficientes Estandarizados } & \multirow[b]{2}{*}{ Sig } \\
\hline & $B$ & Error Estándar & $t$ & \\
\hline Relaciones & -.74 & .39 & -3.12 & .03 \\
\hline Desarrollo & -.35 & .81 & -1.01 & .36 \\
\hline Estabilidad & -.03 & .88 & -.09 & .93 \\
\hline
\end{tabular}

* La variable dependiente es la puntuación total de la Escala de Ideación Suicida.

\section{Discusión}

El entorno familiar es una variable relevante en la intervención con adolescentes con ideación suicida. Este estudio se ha dirigido a conocer con mayor precisión cuáles son los elementos del funcionamiento familiar relacionados con el riesgo suicida en población adolescente con rasgos de personalidad límite. Los resultados señalan la importancia de la dimensión de Relaciones en la familia como factor de riesgo de suicidio en adolescentes con rasgos de personalidad límite, que hace referencia a la calidad de la comunicación familiar. Estos resultados son coherentes con los hallados en el estudio de Xing et al. (2010), donde la comunicación familiar disfuncional se relacionaba con mayores niveles de suicidio en adolescentes.

De todas las subescalas, los resultados apuntan a la influencia específica de la subescala de Expresividad, que se relaciona con el grado de libre expresión emocional en la familia, y a la subescala Social-Recreativa. Estos resultados apuntan a que menores niveles de expresión emocional en la familia se relacionarían con mayor riesgo de suicidio en los adolescentes con rasgos límite de personalidad, y que la participación conjunta en actividades sociales y de ocio disminuye el riesgo de suicido. En contraposición a lo esperado según estudios previos, la subescala Cohesión, relacionada con el apoyo familiar, no presentó una relación significativa con la Ideación Suicida. En consonancia a los resultados de nuestro estudio, Lipschitz, Yen, Weinstock, y Spirito (2012) analizaron la relación entre el funcionamiento familiar percibido por los adolescentes hospitalizados por ideación suicida (evaluado a través de la subescala de funcionamiento general del Dispositivo de Evaluación familiar, FAD y la Escala de Ideación Suicida de Beck). Los resultados mostraron una relación entre peor funcionamiento familiar y mayor ideación suicida.

Por tanto, incluir a la familia en el tratamiento, enfocándose en mejorar la comunicación y la expresión emocional de los miembros y la participación en actividades sociales y recreativas, podría tener efectos en la reducción del riesgo suicida de adolescentes. De acuerdo con Brent et al. (2013), los programas preventivos del suicidio en adolescentes apuntan a la inclusión de la familia como variable implicada en el éxito de las intervenciones. Cuando se incluye a las familias en el tratamiento de los adolescentes suicidas, las recaídas se distancian en el tiempo y el bienestar de la familia aumenta (Dixon et al., 2001). 
Uno de los aspectos controvertidos de esta línea de investigación es la bidireccionalidad de la relación entre el clima familiar y la ideación suicida. Sin embargo, los resultados de estudios que evalúan la eficacia de las intervenciones familiares para reducir el comportamiento suicida en adolescentes sugieren lo contrario. Pineda, Mark, y Dadds (2013) evaluaron la efectividad de una intervención familiar para reducir los comportamientos suicidas en adolescentes no hospitalizados de forma longitudinal, a los 3 y a los 6 meses. El tratamiento demostró ser eficaz para mejorar el funcionamiento familiar y reducir el comportamiento suicida, comparado con la intervención que no incluía a la familia. Los beneficios se mantuvieron en el seguimiento. Los resultados demostraron que el cambio en el comportamiento suicida de los adolescentes estaba mediado por los cambios en el funcionamiento familiar. En consonancia con estos resultados, Diamond et al. (2010) realizaron un estudio con adolescentes suicidas en el que comparaban un grupo que recibía sólo tratamiento para los hijos con uno que además incluía a los padres en el tratamiento. Los resultados de este estudio mostraron un papel mediador de la inclusión de la familia en la reducción del comportamiento suicida en los adolescentes tres meses después del tratamiento.

La relevancia del presente estudio recae en la posibilidad de influir en factores modificables del riesgo suicida en población adolescente con rasgos de personalidad límite. El estudio tiene limitaciones, ya que su carácter transversal limita poder establecer conclusiones acerca de la causalidad o la dirección de la influencia de unas variables sobre otras. Otra de las limitaciones es el tamaño muestral, por lo que la falta de potencia podría haber ensombrecido algunas relaciones existentes por no ser significativas.

Los resultados obtenidos sugieren que las variables familiares relativas a la comunicación y la expresión de emociones son un importante objetivo de tratamiento para reducir el riesgo suicida en adolescentes. Ciertos programas de tratamiento desarrollados para mejorar el manejo y la expresión emocional de padres de adolescentes con rasgos de personalidad límite, como el desarrollado por la Unidad de Adolescentes del Hospital Gregorio Marañón (programa Familias al Límite), deberán ser evaluados para evaluar la eficacia de este tipo de intervenciones en la reducción del riesgo suicida en esta población.

\section{Conflicto de intereses}

Los autores de este trabajo declaran que no existe conflicto de intereses.

\section{Financiación}

Este proyecto ha sido financiado con medios propios.

\section{Agradecimientos}

A todos los que han participado en alguna fase del estudio favoreciendo que haya salido adelante.

\section{Referencias}

American Psychiatric Association. (2013). Diagnostic and Statistical Manual of Mental Disorders: DSM-5. Washington, DC: American Psychiatric Association.

Barksdale, C. L., Walrath, C. M., Compton, J. S., \& Goldston, D. B. (2009). Caregiver strain and youth suicide attempt: Are they related? Suicide \& Life-threatening Behavior, 39(2), 152-160. doi:10.1521/suli.2009.39.2.152

Beck, A. T., Kovacs, M., \& Weissman, A. (1979). Assessment of suicidal intention: The Scale for Suicide Ideation. Journal of Consulting and Clinical Psychology, 47(2), 343-352. doi: 10.1037/0022-006X.47.2.343

Brent, D. A., McMakin, D. L., Kennard, B. D., Goldstein, T. R., Mayes, T. L., \& Douaihy, A. B. (2013). Protecting Adolescents from Self-Harm: A Critical Review of Intervention Studies. Journal of the American Academy of Child and Adolescent Psychiatry, 52(12), 1260-1271. doi: 10.1016/j. jaac.2013.09.009

Chambers, W., Puig-Antich, J., Hirsch, M., Paez, P., Ambrosini, P. J., Tabrizi, M. A., \& Davies, M. (1985). The assessment of affective disorders in children and adolescents by semi-structured interview. Test-retest reliability of the schedule for affective disorders and schizophrenia for school-age children, present episode version. Archives of General Psychiatry, 42(7), 696-702. doi:10.1001/archpsyc.1985.01790300064008

Compton, M. T., Thompson, N. J., \& Kaslow, N. J. (2005). Social environment factors associated with suicide attempt among low-income African Americans: The protective role of family relationships and social support. Social Psychiatry and Psychiatric Epidemiology, 40(3), 175-185. doi: 10.1007/s00127-005-0865-6

Consoli, A., Cohen, D., Bodeau, N., Guilé, J. M., Mirkovic, B., Knafo, A., ... Gérardin, P. (2015). Risk and protective factors for suicidality at 6-month follow-up in adolescent inpatients who attempted suicide: An exploratory model. Canadian Journal of Psychiatry, 60(2 Suppl1), S27-S36.

Conway, C. C., Hammen, C., \& Brennan, P. A. (2015). Adolescent precursors of adult Borderline Personality Pathology in a high-risk community sample. Journal of Personality Disorders, 29(3), 316-333. doi: 10.1521/ pedi_2014_28_158

Diamond, G., Wintersteen, M., Brown, G., Diamond, G., Gallop, R., Shelef, K., \& Levy S. (2010). Attachment-based family therapy for adolescents with suicidal ideation: a randomized controlled trial. Journal of American Academy Child and Adolescent Psychiatry, 49(2), 122-131. doi: 10.1037/23290382.1.S.91

Dixon, L., McFarlane, W., Lefley, H., Lucksted, A., Cohen, M., Falloon, I., .... Sondheimer, D. (2001). Evidence-based practices for services to families of people with psychiatric disabilities. Psychiatry Services, 52(7), 903-910. doi: 10.1176/appi.ps.52.7.903

First, M. B., Gibbon, M., Spitzer, R. L., Williams, J. B.W., \& Benjamin, L. S. (1997). Entrevista Clínica Estructurada para los Trastornos de Personalidad del Eje II del DSM-IV. Barcelona: Masson.

Flouri, E., Buchanan, A., \& Bream, V. (2002). Adolescents' perceptions of their fathers' involvement: Significance to school attitudes. Psychology in Schools, 3, 575-582. doi: 10.1002/pits. 10055

Fulginiti, A., Rice, E., Hsu, H., Rhoades, H., \& Winetrobe, H. (2016). Risky Integration. Crisis, the Journal of Crisis Intervention and Suicide Prevention, 37(3), 184-193. doi: 10.1027/0227-5910/a000374

Gould, M. Greenberg, T., Velting, D. M., \& Shaffer, D. (2003). Youth suicide risk and preventive intervention: a review of the past 10 years. Journal of the American Academy of Child and Adolescence Psychiatry, 42(4), 386405. doi: 10.1097/01.CHI.0000046821.95464.CF 
Groholt, B., Ekeberg, O., Wichstrom, L., \& Haldorsen, T. (2000). Young suicide attempters: a comparison between a clinical and an epidemiological sample. Journal of the American Academy of Child and Adolescence Psychiatry, 39, 868-875. doi: 10.1097/00004583-200007000-00015

Gutierrez, P. M. (2006). Integratively assessing risk and protective factors for adolescent suicide. Suicide and Life-Threatening Behavior, 36, 129-135. doi: 10.1521/suli.2006.36.2.129

Harris, T., \& Molock, S. (2000). Cultural orientation, family cohesion, and family support in suicide ideation and depression among African American college students. Suicide and Life-Threatening Behavior, 30, 341-353. doi: 10.1111/j.1939-0025.2010.01078.x

Insel, P. M., \& Moos, R. H. (1974). Psychological environments: Expanding the scope of human ecology. American Psychologist, 29(3), 179-188. doi: 10.1037/h0035994

Kaess, M., Brunner, R., \& Chanen, A. (2014). Borderline personality disorder in adolescence. Pediatrics.134(4), 782-793. doi: 10.1542/peds.2013-3677

King, C., Segal, H., Kaminski, K., Naylor, M., Ghaziuddin N., \& Radpour, L. (1995). A prospective study of adolescent suicidal behavior following hospitalization. Suicide and Life-Threatening Behavior, 25, 327-338. doi: 10.1080/15374416.2013.836454

Lenzenweger, M. F., Lane, M. C., Loranger, A. W., \& Kessler, R. C. (2007). DSM-IV Personality Disorders in the National Comorbidity Survey Replication (NCS-R). Biological Psychiatry, 62, 553-564. doi: 10.1016/j. biopsych.2006.09.019

Linehan, M. (1993). Cognitive Behavioral Treatment of Borderline Personality Disorder. New York: Guilford Press.

Lipschitz, J., Yen, S., Weinstock, L., \& Spirito, A. (2012). Adolescent and caregiver perception of family functioning: relation to suicide ideation and attempts. Psychiatry Research, 200(2-3), 400-403. doi: 10.1016/j. psychres.2012.07.051

Lucksted, A., Stewart, B., \& Forbes, C. (2008). Benefits and changes for family to family graduates. American Journal of Community Psychology, (1-2), 154-166. doi: 10.1007/s10464-008-9195-7

Machell, K., Rallis, B., \& Esposito-Smythers, C. (2016). Family environment as a moderator of the association between anxiety and suicidal ideation. Journal of Anxiety Disorders, 40, 1-7. doi: 10.1016/j.janxdis.2016.03.002.

McDonell, M., Short, R., Berry, C., \& Dyck, D. G. (2003). Burden in schizophrenia caregivers: impact of family psychoeducation and awareness of patient suicidality. Family Process, 42(1), 91-103. doi: 10.1111/j.15455300.2003.00091.x

Meadows, L., Kaslow, N., Thompson, M., \& Jurkovic G. (2005). Protective factors against suicide attempt risk among African American women experiencing intimate partner violence. American Journal of Community Psychology, 36(1-2), 109-121. doi: 10.1007/s10464-005-6236-3

Miller, A. L., Muehlenkamp, J. J., \& Jacobson, C. M. (2008). Fact or fiction: diagnosing borderline personality disorder in adolescents. Clinical Psychology Review, 28(6), 969-981. doi: 10.1016/j.cpr.2008.02.004

Miller, A. L., Rathus, J. H., \& Linehan, M. M. (2007) Dialectical Behavior Therapy with Suicidal Adolescents. New York, NY: The Guilford Press.

Moos, R. H. (1974). The social climate scales: an overview. Palo Alto: Consulting Psychologists Press.

Organización Mundial de la Salud \& Organización Panamericana de la Salud (邓2014)凶. Prevención del suicidio: un imperativo global. Organización Panamericana de la Salud.

Pineda, J., Mark, R., \& Dadds, M. (2013). Family intervention for adolescents with suicidal behaviour: A randomized controlled trial and mediation analysis. Journal of the American Academy of Child and Adolescent Psychiatry, 52(8), 851-862. doi: 10.1016/j.jaac.2013.05.015
Raphael, H., Clarke, G., \& Kumar, S. (2006). Exploring parents' responses to their child's deliberate self-harm. Health Education, 106(1), 9-20. doi: $10.1108 / 09654280610637166$

Seisdedos, N., Victoria de la Cruz, M., \& Cordero, A. (1989). Escalas de Clima Social (FES). Madrid: TEA Ediciones.

Skegg, K. (2005). Self-harm. The Lancet, 366(9495), 1471-1483. doi: 10.1016/ S0140-6736(05)67600-3

Tyrer, P., Crawford, M., \& Mulder, R. (2011). Reclassifying personality disorders. The Lancet, 377(9780):1814-1815. doi: 10.1016/S01406736(10)61926-5

Xing, X., Tao, F., Wan, Y., Xing, C., Qi, X., Hao, J., Su, P., Pan, H., \& Huang, L. (2010). Family factors associated with suicide attempts among Chinese adolescent students: A national cross-sectional survey. Journal of Adolescent Health, 46, 592-599. doi: 10.1016/j.jadohealth.2009.12.006

Yen, S., Gagnon, K., \& Spirito, A. (2013). Borderline personality disorder in suicidal adolescents. Personality \& Mental Health, (2): 89-101. doi: 10.1002/pmh.1216

Zimmerman, M., Rothschild, L., \& Chelminski, I. (2005). The prevalence of DSM-IV personality disorders in psychiatric outpatients. American Journal of Psychiatry, 162(10), 1911-1918. doi: 10.1176/appi.ajp.162.10.1911 